OPEN ACCESS

Edited by:

Klaus Pantel,

University Medical Center

Hamburg-Eppendorf, Germany

Reviewed by:

Juçara Gastaldi Cominal,

Institut Imagine, France

Michal Mego,

Campus Bio-Medico University, Italy

*Correspondence:

Pawel Mach

pawel.mach@uk-essen.de

Specialty section:

This article was submitted to

Molecular and Cellular Oncology,

a section of the journal

Frontiers in Oncology

Received: 05 June 2021 Accepted: 13 October 2021 Published: 29 October 2021

Citation:

Mach P, Kimmig R, Kasimir-Bauer S and Buderath $P$ (2021) Association of

Soluble B7-H4 and Circulating

Tumor Cells in Blood of Advanced

Epithelial Ovarian Cancer Patients.

Front. Oncol. 11:721067.

doi: 10.3389/fonc.2021.721067

\section{Association of Soluble B7-H4 and Circulating Tumor Cells in Blood of Advanced Epithelial Ovarian Cancer Patients}

\author{
Pawel Mach ${ }^{*}$, Rainer Kimmig, Sabine Kasimir-Bauer and Paul Buderath \\ Department of Gynecology and Obstetrics, University Hospital Essen, Essen, Germany
}

Introduction: Epithelial ovarian cancer (EOC) is the deadliest gynecologic malignancy worldwide. Reliable predictive biomarkers are urgently needed to estimate the risk of relapse and to improve treatment management. Soluble immune-checkpoints in EOC are promising molecules serving as prognostic biomarkers accessible via liquid biopsy. We thus, aimed at elucidating the role of sB7-H4 in EOC.

Material and Methods: We analyzed soluble serum B7-H4 (sB7-H4) using ELISA and circulating tumor cells (CTCs) in blood applying the AdnaTest OvarianCancer in 85 patients suffering from advanced EOC. Findings were correlated with clinical parameters as well as survival data.

Results: sB7-H4 was detectable in $14.1 \%$ patients, CTCs in $32.9 \%$ patients and simultaneous presence of CTCs and $\mathrm{sB} 7-\mathrm{H} 4$ was found in $7 \%$ patients, respectively. Although no association between sB7-H4 and CTC could be documented, each of them served as independent predictive factors for overall survival (OS).

Conclusion: sB7-H4 and CTCs are independent prognostic biomarkers for impaired survival in EOC. As they are easily accessible via liquid biopsy, they may be of potential benefit for the prediction of therapy response and survival for EOC patients.

Keywords: epithelial ovarian cancer, sB7-H4, circulating tumor cells (CTC), tumor micro-environment, biomarker

\section{INTRODUCTION}

Epithelial ovarian cancer (EOC) is the deadliest gynecologic malignancy worldwide (1). In Germany, EOC is responsible for 3.2\% of all malignant neoplasms and $5.3 \%$ of all cancer deaths (2). Despite a high response rate for initial therapy, most patients eventually relapse (3), leading to a relative 5-year survival of only $41 \%$ in 2014 (2). Reliable predictive biomarkers are urgently needed to estimate the risk of relapse and improve treatment management. In this regard, it has already been demonstrated that the characterization of disseminated tumor cells (DTCs) in the bone marrow (BM) and circulating tumor cells (CTCs) in peripheral blood has identified stem cell like DTCs and CTCs, tumor cells in epithelial-mesenchymal transition (EMT) as well as resistant cells, all associated with a worse outcome and clinical platinum resistance (4-7). Interestingly, we were able to show that immunotherapy based on the intraperitoneal trifunctional bispecific antibody 
Catumaxomab was successful in the elimination of DTCs and CTCs in patients with advanced EOC (8). Thus, the tumor microenvironment might play a crucial role in tumor control and tumorigenesis of these patients.

In recent years, the role of the immune system in EOC has gathered increasing attention. Several mechanisms of immuneescape have been identified in malignant tumors, including the aberrant expression of immune costimulatory ligands, such as those of the B7 family, namely programmed cell-death protein 1 (PD-1) (also known as B7-H1) and its ligands, programmed celldeath ligand 1 and 2 (PD-L1 and PD-L2). In our recent study, we show that decreased serum levels of PD-L2 were associated with poor survival and platinum-resistance, as well as the presence of DNA excision repair protein ERCC+CTC, indicating the significance of the organism's immune-response for the prevention of tumor-cell spread and the efficacy of platinumbased therapy (9).

B7-H4 is another member of the B7-family, which is highly expressed in various types of neoplasms including ovarian, endometrial, or breast cancer (BC). Its expression correlates with the progression of the disease and a poor prognosis in many cases $(10,11)$. Moreover, Liang et al. show that B7-H4 is more often expressed in high-grade serous EOC (12). Evaluating a serum-based, multi parametric biomarker panel in OC patients, Oikonomopoulou et al. found that high levels of B7$\mathrm{H} 4$ were associated with worse overall survival (13). B7-H4 has a membrane-bound and soluble form (sB7-H4), which has also been detected in the serum of patients suffering from cancer with its expression closely related to progression and prognosis (14). However, the source and function of sB7-H4 are still unknown.

The hypothesis that immune cells in the bloodstream can expand the metastatic potential of CTCs has recently been confirmed in BC (15). Based on these findings, we hypothesize that simultaneous measurements of CTCs and sB7-H4 could help to better understand the complex interactions between the tumor microenvironment and the immune response in EOC. This study aimed to investigate the serum levels of sB7-H4 and CTCs in patients with advanced EOC and to analyze its relationship to prognosis and clinicopathological features.

\section{MATERIAL AND METHODS}

\section{Cohort Characteristics}

We analyzed data from 85 patients suffering from advanced EOC. All patients underwent primary debulking surgery including hysterectomy, bilateral salpingoovarectomy, omentectomy, peritonectomy and - in the case of macroscopic complete resection - systematic pelvic and paraaortic lymphadenectomy. Adjuvant treatment consisted of six cycles of chemotherapy with carboplatinum AUC5 and paclitaxel 175 $\mathrm{mg} / \mathrm{m}^{2}$ i.v. d1 q3w intravenously. Since 2012, patients presenting at FIGO stage IIIB or higher have been offered an additional adjuvant therapy with the antiangiogenetic antibody Bevacizumab $15 \mathrm{mg} / \mathrm{kg}$ i.v. d1 q3w for a total of 15 months. Patients were diagnosed between 2007 and 2016 at the university hospital in Essen, Germany. Clinical data were acquired from patient charts and the hospital's clinical information system. The study was approved by the local ethics committee of the University of Duisburg-Essen (Essen 05-2870 and 17-7859).

Patients recurring within six months after the end of the adjuvant platinum therapy were considered platinum-resistant. Disease-Free Survival (DFS) was calculated from the date of initial diagnosis to relapse.

An overview of patients' characteristics is given in Table $\mathbf{1}$.

\section{Blood Serum Collection and Measurement of sB7-H4}

Soluble (s) B7-H4 serum levels were analyzed preoperatively using the sandwich Enzyme-Linked Immunosorbent Assay Kit (Cusabio, Cologne, Germany) according to the manual instructions. For ELISA measurement, $100 \mu \mathrm{l}$ undiluted serum samples and control samples were dispensed into wells coated with an antibody specific for B7-H4 and incubated for two hours at $37^{\circ} \mathrm{C}$. Subsequently, after removing any unbound substances, a biotin-conjugated antibody specific for $\mathrm{B} 7-\mathrm{H} 4$ was added to the wells for one hour at $37^{\circ} \mathrm{C}$. After washing three times, $100 \mu \mathrm{L}$ of avidin conjugated Horseradish Peroxidase (HRP) were added for $1 \mathrm{~h}$ at $37^{\circ} \mathrm{C}$. Subsequently, after washing five times, $90 \mu \mathrm{L}$ of substrate solution containing TMB were added for 15-30 minutes at $37^{\circ} \mathrm{C}$, protected from light. Color development was stopped by the addition of $50 \mu \mathrm{l}$ stop solution to each well and the degree of enzymatic turnover of the substrate was investigated by dual-wavelength absorbance measurement at 450 and $620 \mathrm{~nm}$ as a reference wavelength within 5 minutes using an ELISA reader (TECAN, Model Sunrise; Austria GmbH, Grodig, Austria) and the data analysis software Magellan ${ }^{\mathrm{TM}}$ (TECAN, Mannedorf, Switzerland). To quantify the blood serum concentration levels of $\mathrm{B} 7-\mathrm{H} 4$, a non-linear regression model (4-parameter Marquardt) was used with a log/lin type of graph according to the manufacturer's instructions. The observed absorbance was directly proportional to the

TABLE 1 | Clinical data of patients with advanced EOC at the time of primary diagnosis.

\begin{tabular}{|c|c|c|}
\hline & Total $n=85(\%)$ & $\mathrm{p}$-value \\
\hline \multicolumn{3}{|l|}{ FIGO stage } \\
\hline III & $61(71.8)$ & 0.001 \\
\hline IV & 24 (28.2) & \\
\hline \multicolumn{3}{|l|}{ Grading } \\
\hline 1 and 2 & 24 (29.3) & 0.0002 \\
\hline 3 & $58(70.7)$ & \\
\hline \multicolumn{3}{|l|}{ Nodal status } \\
\hline Node negative & $24(37.5)$ & 0.04 \\
\hline Node positive & $40(62.5)$ & \\
\hline \multicolumn{3}{|l|}{ Platinum sensitivity } \\
\hline Sensitive & $50(84.7)$ & 0.0001 \\
\hline Resistant & $23(27.1)$ & \\
\hline \multicolumn{3}{|l|}{ Resection status } \\
\hline Complete resection & $39(48.1)$ & 0.73 \\
\hline Incomplete resection & $42(51.9)$ & \\
\hline \multicolumn{3}{|l|}{ Recurrence } \\
\hline No & $29(37.7)$ & 0.03 \\
\hline Yes & 48 (62.3) & \\
\hline
\end{tabular}


concentration level of sB7-H4 in the samples, which was calculated from the calibration curve. The sB7-H4 serum levels are expressed in $\mathrm{ng} / \mathrm{mL}$ according to the established standard curve (detection range: 7.8 - $500 \mathrm{ng} / \mathrm{mL}$ ). The minimum detectable dose of sB7-H4 was typically less than $1.95 \mathrm{ng} / \mathrm{mL}$. The lower limit of detection (LLD) was defined as the lowest protein concentration that could be differentiated from zero. Intra-assay variation was $<8 \%$, while inter-assay variation was $<10 \%$.

\section{Selection and Detection of CTCs}

The selection, detection, characterization and data evaluation of epithelial CTCs and CTCs in EMT in EOC has been published in detail by our group $(5,16-18)$.

$10 \mathrm{ml}$ blood was collected in EDTA tubes (Sarstedt \& Co.) from each patient and processed within $4 \mathrm{~h}$ for the enrichment of CTCs and subsequent expression analysis according to the Adnatest OvarianCancer (QIAGEN, Hilden, Germany). Briefly, CTCs were immunomagnetically selected using the AdnaTests OvarianCancerSelect and EMT-1Select. Subsequently, RNA was isolated and gene expression analysis was performed by reversetranscription (RT) and multiplex RT-PCR detecting a) EpCAM, MUC-1, and CA-125 (AdnaTest OvarianCancerDetect; ERCC1transcripts were investigated in a separate approach by singleplex RT-PCR) and b) PIK3CA, AKT2 and TWIST (AdnaTest EMT).

All AdnaTests were purchased from QIAGEN (Hilden, Germany). Positivity for each CTC-subtype was defined by the detection of at least one of the transcripts of each marker panel, respectively. A patient was defined as CTC-positive if epithelial CTCs and/or CTCs in EMT were detected.

\section{Statistical Analysis}

The distribution of sB7-H4 in each study group was different from normal. Descriptive statistics were computed and reported as median with interquartile range (IQR) or frequency counts (\%). The differences between two groups were defined by MannWhitney U test. Differences in frequency counts were analyzed using the Chi-squared test. Kaplan-Meier analysis was performed to analyze DFS and overall survival (OS) probabilities. The difference between survival curves was assessed by using the log rank test. Hazard ratios (HR) with corresponding 95\%-confidence intervals (95\%-CI) were calculated by using Cox proportional hazards regression. The Spearman rank correlation coefficient was calculated to examine whether the sB7-H4 levels were monotonically related to CA12-5 serum levels. All analyses were performed using the MedCalc version 17.9.7 (MedCalc Software bvba, Ostend, Belgium).

\section{RESULTS}

The median age of the patients at primary diagnosis was 60 years (37 - 82, SD 11.7). The clinical data of patients included in the study are shown in Table 1. The majority of patients were in Figo stage III $(71.3 \%)$ compared with stage IV (28.2\%). High-grade tumors (G3) were found in $58(70.7 \%)$ patients whereas Grade 1 and 2 in $24(29.3 \%)$ of patients. $40(62.5 \%)$ of patients had positive lymph-nodes and $24(37.5 \%)$ negative nodal-status. When stratifying according to resection status 39 (48.1\%) had complete resection (R0) compared with 42 (51.9\%) patients with incomplete resection. In $48(62.3 \%)$ patients a recurrent disease was diagnosed (Table 1). Among patients with platinumresistant disease 15 (75\%) had positive nodal-status, compared with $5(25 \%)$ with negative nodal-status. In FIGO IV stage 12 (75\%) patients and in FIGO III stage 28 (70\%) had lymph-nodes metastasis. Follow-up was calculated from the time of diagnosis to the date of last follow-up information or death. Median follow-up time was 35.5 months (0 - 139, SD 28.1). Analyzing only patients who had died, medium time to death was 25 months (0 - 75, SD 22.8). Medium DFS was 27 months (0 130, SD 23.9). Patients with an OS $\leq 4$ months were excluded from survival analyses in order to rule out early, surgery related deaths $(n=12)$.

\section{Association of sB7-H4 Concentration Levels, CTCs, and Clinicopathological Characteristics}

sB7-H4 was detectable in 12/85 (14.1\%) patients, CTCs in 28/85 $(32.9 \%)$ patients and simultaneous presence of CTCs and sB7$\mathrm{H} 4$ was found in 6/85 (7\%) patients.

Epithelial CTCs were found in 10/85 (11.7\%) patients and EMT-like CTCs in 18/85 (21.2\%) patients. In 5/85 (5.9\%) patients, expression of both epithelial and EMT-like CTCs subtypes was found. sB7-H4 positivity was significantly more common in patients without confirmed ( $\mathrm{pN} 0$ ) spread to lymph nodes $(25 \%)$ compared to patients with histologically confirmed ( $\mathrm{pN} 1)$ metastases in lymph nodes $(12.5 \%, \mathrm{p}=0.04)$. Moreover, sB7-H4 presence in serum was significantly associated with platinum resistance $(\mathrm{p}=0.03)$. CTCs presence in blood was slightly higher in platin resistant patients but the difference was not significant. There were no differences in sB7-H4 levels in serum according to clinicopathological parameters. Associations between sB7-H4 concentration levels, CTCs and clinicopathological characteristics including FIGO stage, grading, nodal status, sensibility to platin agents and resection status are presented in Table 2.

\section{Impact of sB7-H4 and CTC Presence on Patients' Survival}

Comparison between patients with detectable sB7-H4 serumlevels and no detectable serum levels revealed a statistically significant effect on OS. Patients with detectable sB7-H4 in blood serum showed a significantly shorter survival time than patients with no sB7-H4 detection (HR 4.42, 95\% CI 1.61 12.13; $\mathrm{p}=0.004)$. No effect on DFS could be observed (Figure 1).

Patients with detectable CTCs showed a significantly shorter survival time than patients in whom no CTCs were detected (HR 2.63, 95\% CI 1.28 - 5.39; $\mathrm{p}=0.008)$. No effect on DFS could be observed (Figure 1).

Univariate Cox regression analysis indicated that the presence of sB7-H4 and CTCs influenced the prognosis of our patients. Patients presenting with enhanced sB7-H4 levels and CTCs had 
TABLE 2 | Associations of sB7-H4 blood serum levels, sB7-H4 presence in serum and CTCs to clinicopathological parameters.

\begin{tabular}{|c|c|c|c|c|c|c|}
\hline Total $n=85$ & $\begin{array}{l}\text { sB7-H4 ng/mL levels } \\
\text { in serum (IQR) }\end{array}$ & $p$-value & $\begin{array}{l}\text { sB7-H4 presence } \\
\text { in serum }(\%)\end{array}$ & $p$-value & $\begin{array}{l}\text { CTCs presence } \\
\text { in blood }(\%)\end{array}$ & p-value \\
\hline \multicolumn{7}{|l|}{ FIGO stage } \\
\hline III & 32.89 (5.41 - 71.9) & 0.79 & $8(13.1)$ & 0.19 & $17(27.8)$ & 0.11 \\
\hline IV & $31.05(22.5-44.47)$ & & $6(25)$ & & $11(45.8)$ & \\
\hline \multicolumn{7}{|l|}{ Grading } \\
\hline | and || & 30.37 (22.5 - 92.97) & 0.58 & 4 (16.6) & 0.4 & $11(45.83)$ & 0.7 \\
\hline III & $31.73(5.01-49.29)$ & & $11(18.9)$ & & 17 (29.3) & \\
\hline \multicolumn{7}{|l|}{ Lymph node status } \\
\hline NO & 21.79 (6.2 - 31.73) & 0.24 & $6(25)$ & 0.04 & $3(12.5)$ & 0.18 \\
\hline N1 & $44.58(17.49-87.6)$ & & $5(12.5)$ & & $16(40)$ & \\
\hline \multicolumn{7}{|l|}{ Platinum sensitivity } \\
\hline Sensitive & $33.48(4.98-47.76)$ & 0.25 & $8(16)$ & 0.03 & $15(30)$ & 0.08 \\
\hline Resistant & $31.05(21.08-82.23)$ & & $6(66.6)$ & & $13(56.5) 23$ & \\
\hline \multicolumn{7}{|l|}{ Resection status } \\
\hline Complete resection & $25.72(5.41-41.28)$ & 0.43 & $8(20.5)$ & 0.4 & $10(25.6)$ & 0.15 \\
\hline Incomplete resection & $44.58(22.5-82.23)$ & & $6(14.3)$ & & $17(40.47)$ & \\
\hline
\end{tabular}

Comparisons between sB7-H4 serum levels were analyzed using Mann-Whitney $U$ test, sB7-H4- and CTC-presence using chi-square test.

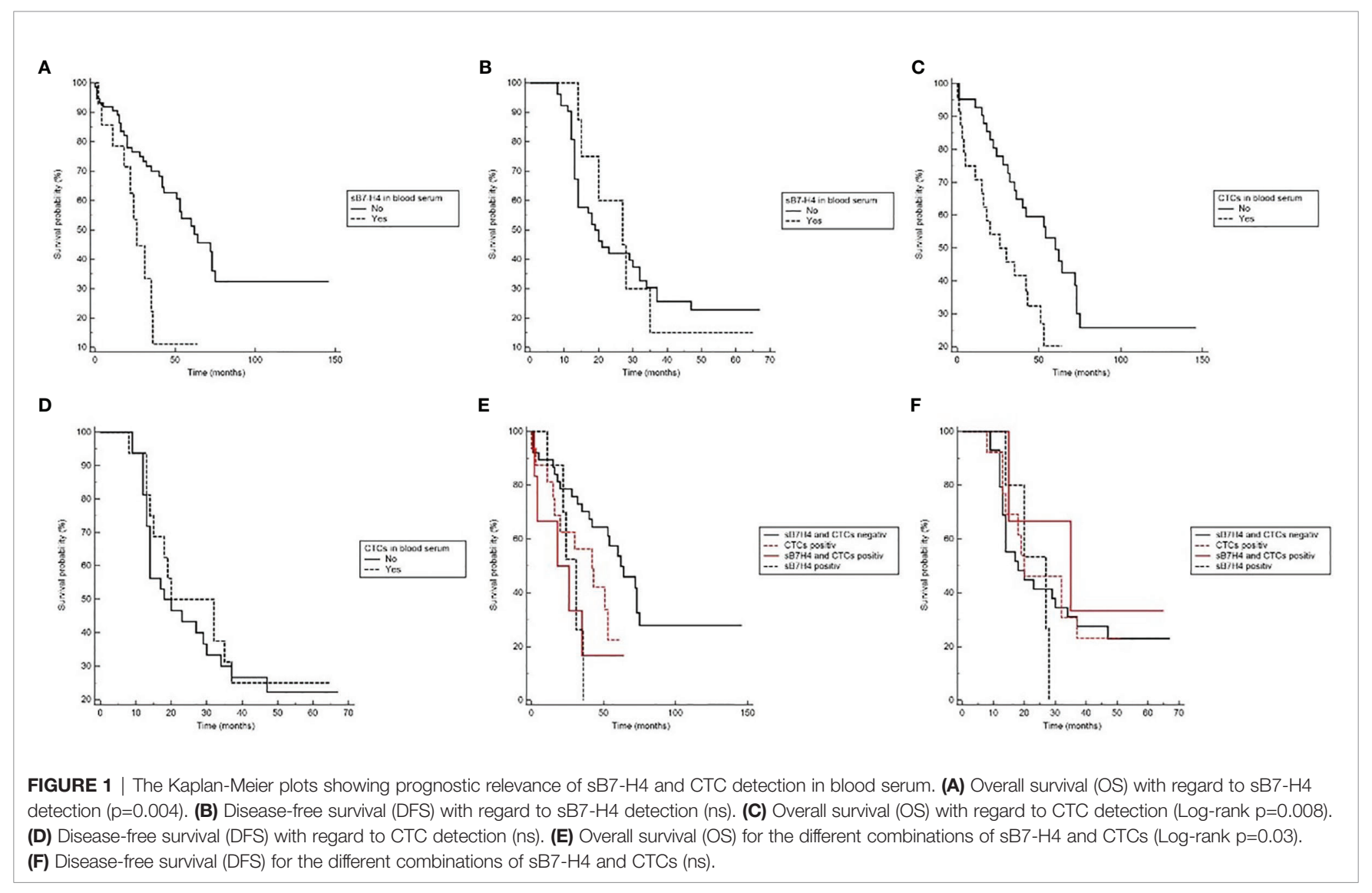

a significantly worse OS in comparison with those patients with no detectable sB7-H4 and CTCs (HR 2.8, 95\% CI 11.34 - 5.82; $\mathrm{p}=0.006$ and HR 2.32, 95\% CI $1.22-4.41 ; \mathrm{p}=0.01$, respectively). The clinical factors associated with prognosis including platinum-sensitivity, resection status, FIGO stadium and lymph node status also correlated with OS, however, apart from platinum-sensitivity, no effect on DFS could be observed (Table 3 ).
Multivariate Cox regression analysis including resection status, FIGO stage, grade, lymph node status, platinumsensitivity, CTC detection and $\mathrm{sB} 7-\mathrm{H} 4$ presence indicated that sB7-H4 and CTCs presence were independent predictive factor for OS (HR 3.57, 95\% CI 1.23 - 10.36; $\mathrm{p}=0.02$ and HR 4.31, 95\% CI 1.53 - 12.17; $\mathrm{p}=0.006$, respectively). sB7-H4 or CTC presence did not significantly influence the adjusted hazard on developing recurrent disease (Table 4). 
TABLE 3 | Results of univariate Cox regression analysis of patients with advanced EOC.

\begin{tabular}{|c|c|c|c|c|}
\hline & \multicolumn{2}{|l|}{ os } & \multicolumn{2}{|l|}{ DFS } \\
\hline & HR (95\% Cl) & p-value & HR (95\% Cl) & p-value \\
\hline \multicolumn{5}{|c|}{$\mathrm{sB} 7-\mathrm{H} 4$ in serum } \\
\hline no & 1 & 0.006 & 1 & 0.82 \\
\hline yes & $2.8(1.34-5.82)$ & & $0.9(0.38-2.14)$ & \\
\hline \multicolumn{5}{|c|}{ CTCs in blood } \\
\hline no & 1 & 0.01 & 1 & 0.4 \\
\hline yes & $2.32(1.22-4.41)$ & & $0.85(0.42-1.69)$ & \\
\hline \multicolumn{5}{|c|}{ Residual tumor } \\
\hline no & 1 & 0.01 & 1 & 0.16 \\
\hline yes & $2.13(1.17-3.88)$ & & $1.54(0.84-2.82)$ & \\
\hline \multicolumn{5}{|l|}{ FIGO stage } \\
\hline III & 1 & 0.12 & 1 & 0.65 \\
\hline IV & $1.63(0.88-3.04)$ & & $0.85(0.42-1.72)$ & \\
\hline \multicolumn{5}{|l|}{ Grade } \\
\hline$|/| \mid$ & 1 & 0.78 & 1 & 0.79 \\
\hline III & 1.09 (0.59 - 2.03) & & $1.09(0.56-2.08)$ & \\
\hline \multicolumn{5}{|c|}{ Lymph node status } \\
\hline NO & 1 & 0.1 & 1 & 0.47 \\
\hline N1 & 1.95 (0.87 - 4.39) & & $1.35(0.6-3.03)$ & \\
\hline \multicolumn{5}{|c|}{ Platinum sensitivity } \\
\hline Sensitive & 1 & $<0.0001$ & 1 & 0.03 \\
\hline Resistant & $7.03(4.1-12.08)$ & & $10.8(1.26-92.74)$ & \\
\hline
\end{tabular}

$\mathrm{Cl}$, confidence interval; HR, hazard ratio.

TABLE 4 | Effect of sB7-H4 and CTCs presence on OS and DFS after correcting for unfavorable prognostic factors in cox proportional hazards regression model.

\begin{tabular}{|c|c|c|c|c|}
\hline & \multicolumn{2}{|l|}{ os } & \multicolumn{2}{|l|}{ DFS } \\
\hline & HR (95\% Cl) & $p$-value & HR $(95 \% \mathrm{Cl})$ & p-value \\
\hline \multirow{2}{*}{\multicolumn{5}{|c|}{$\begin{array}{l}\text { sB7-H4 in } \\
\text { serum }\end{array}$}} \\
\hline & & & & \\
\hline no & 1 & \multirow[t]{2}{*}{0.02} & 1 & \multirow[t]{2}{*}{0.74} \\
\hline yes & $3.57(1.23-10.36)$ & & $1.3(0.27-6.08)$ & \\
\hline \multicolumn{5}{|c|}{ CTCs in blood } \\
\hline no & 1 & \multirow[t]{2}{*}{0.006} & 1 & \multirow[t]{2}{*}{0.35} \\
\hline yes & $4.31(1.53-12.17)$ & & $0.59(0.19-1.78)$ & \\
\hline \multicolumn{5}{|c|}{ Residual tumor } \\
\hline no & 1 & \multirow[t]{2}{*}{0.3} & 1 & \multirow[t]{2}{*}{0.65} \\
\hline yes & $1.53(0.61-3.83)$ & & $1.27(0.45-3.59)$ & \\
\hline \multicolumn{5}{|l|}{ FIGO stage } \\
\hline III & 1 & \multirow[t]{2}{*}{0.16} & 1 & \multirow[t]{2}{*}{0.86} \\
\hline IV & $0.47(0.16-1.36)$ & & $0.89(0.23-3.4)$ & \\
\hline \multicolumn{5}{|l|}{ Grade } \\
\hline$|/| \mid$ & 1 & \multirow[t]{2}{*}{0.22} & 1 & \multirow[t]{2}{*}{0.4} \\
\hline III & $0.58(0.25-1.38)$ & & $1.5(0.57-3.93)$ & \\
\hline \multicolumn{5}{|l|}{$\begin{array}{l}\text { Lymph node } \\
\text { status }\end{array}$} \\
\hline NO & 1 & \multirow[t]{2}{*}{0.58} & 1 & \multirow[t]{2}{*}{0.73} \\
\hline N1 & $1.31(0.5-3.4)$ & & $1.21(0.41-3.54)$ & \\
\hline \multicolumn{5}{|l|}{$\begin{array}{l}\text { Platinum } \\
\text { sensitivity }\end{array}$} \\
\hline Sensitive & 1 & $<0.0001$ & 1 & $<0.0001$ \\
\hline Resistant & $7.24(3.03-17.33)$ & & $59.1(4.52-771.97)$ & \\
\hline
\end{tabular}

$\mathrm{Cl}$, confidence interval; HR, hazard ratio.

\section{sB7-H4 and CTCs}

22 out of 59 patients (37.2\%) with normal sB7-H4 levels had detectable CTCs, whereas in the group of patients with detectable sB7-H4 levels, 6 out of 12 (50\%) patients were positive for CTCs. However, this effect failed to reach statistical significance $(\mathrm{p}=$ 0.41 ). An overview on the presence of $\mathrm{sB} /-\mathrm{H} 4$ and CTCs is given in (Table 5).

There were no differences in sB7-H4 serum levels between patients harboring CTCs (median 31.05, IQR13.64-63.46) or not (median 27.29, IQR 3.76-50.83) ( $\mathrm{p}=0.69)$.

Patients with detectable sB7-H4 levels and CTCs showed a significantly shorter survival time than patients with normal sB7H4 levels and no detectable CTCs $(\mathrm{p}=0.03)$. No effects on DFS could be observed (Figure 1).

\section{DISCUSSION}

Our study found that detection of sB7-H4 and CTCs in blood was associated with a worse prognosis of patients with advanced EOC. However, there was no association between sB7-H4 and CTC presence. Current literature supports that high expression of B7H4 was negatively correlated with survival outcome in OC, suggesting that $\mathrm{B} 7-\mathrm{H} 4$ plays an essential role in poor prognosis (16). However in a meta-analysis from Ye et al., membrane-bound B7-H4 was not related to patients' clinicopathologic characteristics (19). These results are consistent with our findings. However, it is not clear whether serum sB7-H4 levels reflect the expression of B7-H4 in tumor tissue. Similarly to the previous study for sB7-H4 and EOC (13), we found that serum sB7-H4 predicted a worse prognosis (being a predictive factor for OS), independent of clinical parameters known for worse outcomes.

In our study, patients with detectable sB7-H4 and CTCs in blood showed significantly worse survival. The function and source of sB7-H4 are still unknown but some authors have demonstrated that sB7-H4 secretion is promoted under inflammatory conditions (20-22) and in EOC, B7-H4 has been associated with a pro-inflammatory tumor microenvironment (23). On the other hand, the neutrophile-derived inflammatory response was shown to interact with CTCs expanding their metastatic potential (24). These data could be a possible explanation of the worse prognosis of EOC patients showing the simultaneous presence of sB7-H4 and CTCs in the bloodstream.

In our study sB7-H4 was found more in patients without confirmed spread to lymph-nodes, which could suggest its association with better outcome. This fact may be explained due to early way of dissemination in EOC, which is primary into

TABLE 5 | Presence of sB7-H4 and CTCs in blood serum in patients with advanced EOC.

\begin{tabular}{lcc}
\hline & $\begin{array}{c}\text { sB7-H4 } \\
\text { detectable }\end{array}$ & $\begin{array}{c}\text { sB7-H4 not } \\
\text { detectable }\end{array}$ \\
\hline CTCs detectable in blood serum & 6 & 22 \\
CTCs not detectable in blood serum & 6 & 37
\end{tabular}


the peritoneal cavity and not into lymph-nodes. On the contrary, $\mathrm{sB} 7-\mathrm{H} 4$ presence was associated with platinum resistance, which is one of the most important factors connected with worse prognosis. These findings suggest that the tumor biology, rather than tumor load are responsible for sB7-H4 presence in advanced EOC.

The interaction between tumor cells and their microenvironment plays a crucial role in cancer development (25). Current data on the relationship between T-Cell regulators and CTCs in EOC are limited. However, strong evidence suggests a direct interaction between CTCs and the immune system in the peripheral blood. Mego et al. showed that patients with inflammatory BC harboring CTCs had an impaired adaptive immunity (26). In the current study, both, the presence of CTCs and $\mathrm{sB} 7-\mathrm{H} 4$ were associated with a worse prognosis. Postulating a function of B7-H4 as part of the tumor's immune-escape mechanisms, it seems plausible that an increased expression of the immune-checkpoint molecule increases the risk of tumor spread to the blood, indicating poor outcome. This reflects higher sB7-H4 levels in patients with positive CTCs. This is in line with our recent findings analyzing soluble PD-L1 and PD-L2 in EOC patients (9). However, we did not find any association between sB7-H4 and CTCs presence in serum. Keeping in mind that sB7-H4 and CTCs were detectable in only 15 and 28 patients, respectively, this lacking association might well be attributable to the small sample size. Nevertheless, the potential association between CTCs and soluble and membrane $\mathrm{T}$-cells regulators should be further investigated since checkpoint-inhibitors showed promising results in therapy management of advanced EOC $(27,28)$.

Expression of immune-checkpoints on CTCs can be used as a prognostic indicator and to monitor and evaluate the efficacy of immune checkpoint inhibitors (29-33). For instance, in patients diagnosed with breast cancer, PD-L1-positive CTCs have a prognostic predictive value (33). Our data suggest that the simultaneous detection of sB7-H4 and CTCs in blood serum might have prognostic value.

The role of immune-checkpoint inhibition in the therapy of advanced EOC has been intensively investigated in recent years. It was reported that $\mathrm{B} 7-\mathrm{H} 4 / \mathrm{CD} 3$-bispecific antibodies might be a therapeutic agent against B7-H4-expressing tumors (34). One of the biggest issues concerning checkpoint-inhibitor therapy is the identification of factors predicting therapy response. Therefore, it is crucial to identify markers that can predict the possible response to this treatment option. The presence of sB7-H4

\section{REFERENCES}

1. Mirza MR, Monk BJ, Herrstedt J, Oza AM, Mahner S, Redondo A, et al. Niraparib Maintenance Therapy in Platinum-Sensitive, Recurrent Ovarian Cancer. N Engl J Med (2016) 375(22):2154-64. doi: 10.1056/NEJMoa1611310

2. Barnes B, Kraywinkel K, Nowossadeck E, et al. Bericht Zum Krebsgeschehen in Deutschland In: Robert Koch-Institute. (2016).

3. Heintz AP, Odicino F, Maisonneuve P, Quinn MA, Benedet JL, Creasman WT, et al. Carcinoma of the Ovary. FIGO 26th Annual Report on the Results of Treatment in Gynecological Cancer. Int J Gynaecol Obstet (2006) 95 Suppl 1:S161-92. doi: 10.1016/S0020-7292(06)60033-7 could be a predictive marker for immunotherapy, targeting Tcells as shown by Azuma $(35,36)$.

There are some limitations to this study. Unfortunately, we do not have results for $\mathrm{B} 7-\mathrm{H} 4$ on tumor tissue for comparison studies, which should be addressed in future work. In addition, we presented a retrospective study with limited sample size. Nevertheless, our data suggest that sB7-H4 and CTCs should be considered potentially useful markers, predicting the prognosis in advanced EOC. A deeper understanding of this interaction may provide an opportunity for new therapeutic strategies.

\section{DATA AVAILABILITY STATEMENT}

The raw data supporting the conclusions of this article will be made available by the authors, without undue reservation.

\section{ETHICS STATEMENT}

The studies involving human participants were reviewed and approved by Ethics committee of the University of DuisburgEssen (Essen 05-2870 and 17-7859). The patients/participants provided their written informed consent to participate in this study.

\section{AUTHOR CONTRIBUTIONS}

PM: study design, data acquisition, providing of blood and tissue samples, statistical analysis, and manuscript writing. RK: providing of blood and tissue samples, and manuscript editing. SK-B: study design, data acquisition, laboratory analyses, providing of blood and tissue samples, and manuscript editing. PB: study design, data acquisition, providing of blood and tissue samples, statistical analysis, and manuscript writing. All authors contributed to the article and approved the submitted version.

\section{ACKNOWLEDGMENTS}

We gratefully thank the patients and healthy controls for participation in the study and for kindly providing the samples.

4. Giannopoulou L, Kasimir-Bauer S, Lianidou ES. Liquid Biopsy in Ovarian Cancer: Recent Advances on Circulating Tumor Cells and Circulating Tumor DNA. Clin Chem Lab Med (2018) 56(2):186-97. doi: 10.1515/cclm-2017-0019

5. Kuhlmann JD, Wimberger P, Bankfalvi A, Keller T, Scholer S, Aktas B, et al. ERCC1-Positive Circulating Tumor Cells in the Blood of Ovarian Cancer Patients as a Predictive Biomarker for Platinum Resistance. Clin Chem (2014) 60(10):1282-9. doi: 10.1373/clinchem.2014.224808

6. Romero-Laorden N, Olmos D, Fehm T, Garcia-Donas J, Diaz-Padilla I. Circulating and Disseminated Tumor Cells in Ovarian Cancer: A Systematic Review. Gynecologic Oncol (2014) 133(3):632-9. doi: 10.1016/ j.ygyno.2014.03.016 
7. Chebouti I, Kasimir-Bauer S, Buderath P, Wimberger P, Hauch S, Kimmig R, et al. EMT-Like Circulating Tumor Cells in Ovarian Cancer Patients are Enriched by Platinum-Based Chemotherapy. Oncotarget (2017) 8(30):4882031. doi: 10.18632 /oncotarget.16179

8. Wimberger P, Heubner M, Lindhofer H, Jager M, Kimmig R, Kasimir-Bauer S. Influence of Catumaxomab on Tumor Cells in Bone Marrow and Blood in Ovarian Cancer. Anticancer Res (2009) 29(5):1787-91.

9. Buderath P, Schwich E, Jensen C, Horn PA, Kimmig R, Kasimir-Bauer S, et al. Soluble Programmed Death Receptor Ligands sPD-L1 and sPD-L2 as Liquid Biopsy Markers for Prognosis and Platinum Response in Epithelial Ovarian Cancer. Front Oncol (2019) 9:1015. doi: 10.3389/fonc.2019.01015

10. Cui Y, Li Z. B7-H4 is Predictive of Poor Prognosis in Patients With Gastric Cancer. Med Sci Monit (2016) 22:4233-7. doi: 10.12659/MSM.897781

11. Wang L, Yang C, Liu XB, Wang L, Kang FB. B7-H4 Overexpression Contributes to Poor Prognosis and Drug-Resistance in Triple-Negative Breast Cancer. Cancer Cell Int (2018) 18:100. doi: 10.1186/s12935-018-0597-9

12. Liang L, Jiang Y, Chen JS, Niu N, Piao J, Ning J, et al. B7-H4 Expression in Ovarian Serous Carcinoma: A Study of 306 Cases. Hum Pathol (2016) 57:1-6. doi: 10.1016/j.humpath.2016.06.011

13. Oikonomopoulou K, Li L, Zheng Y, Simon I, Wolfert RL, Valik D, et al. Prediction of Ovarian Cancer Prognosis and Response to Chemotherapy by a Serum-Based Multiparametric Biomarker Panel. Br J Cancer (2008) 99 (7):1103-13. doi: 10.1038/sj.bjc.6604630

14. Zhang C, Li Y, Wang Y. Diagnostic Value of Serum B7-H4 for Hepatocellular Carcinoma. J Surg Res (2015) 197(2):301-6. doi: 10.1016/j.jss.2015.04.034

15. Miklikova S, Minarik G, Sedlackova T, Plava J, Cihova M, Jurisova S, et al. Inflammation-Based Scores Increase the Prognostic Value of Circulating Tumor Cells in Primary Breast Cancer. Cancers (Basel) (2020) 12(5):1134. doi: $10.3390 /$ cancers 12051134

16. Aktas B, Kasimir-Bauer S, Heubner M, Kimmig R, Wimberger P. Molecular Profiling and Prognostic Relevance of Circulating Tumor Cells in the Blood of Ovarian Cancer Patients at Primary Diagnosis and After Platinum-Based Chemotherapy. Int I gynecological Cancer Off J Int Gynecological Cancer Soc (2011) 21(5):822-30. doi: 10.1097/IGC.0b013e318216cb91

17. Chebouti I, Blassl C, Wimberger P, Neubauer H, Fehm T, Kimmig R, et al. Analysis of Disseminated Tumor Cells Before and After Platinum Based Chemotherapy in Primary Ovarian Cancer. Do stem cell like cells predict prognosis? Oncotarget (2016) 7(18):26454-64. doi: 10.18632/oncotarget.8524

18. Chebouti I, Kuhlmann JD, Buderath P, Weber S, Wimberger P, Bokeloh Y, et al. ERCC1-Expressing Circulating Tumor Cells as a Potential Diagnostic Tool for Monitoring Response to Platinum-Based Chemotherapy and for Predicting Post-Therapeutic Outcome of Ovarian Cancer. Oncotarget (2017) 8 (15):24303-13. doi: 10.18632/oncotarget.13286

19. Ye Y, Wang JJ, Li SL, Wang SY, Jing FH. Does B7-H4 Expression Correlate With Clinicopathologic Characteristics and Survival in Ovarian Cancer?: A Systematic Review and PRISMA-Compliant Meta-Analysis. Med (Baltimore) (2018) 97(32):e11821. doi: 10.1097/MD.0000000000011821

20. Leandersson P, Kalapotharakos G, Henic E, Borgfeldt H, Petzold M, Hoyer-Hansen G, et al. A Biomarker Panel Increases the Diagnostic Performance for Epithelial Ovarian Cancer Type I and II in Young Women. Anticancer Res (2016) 36(3):957-65.

21. Kamimura Y, Kobori H, Piao J, Hashiguchi M, Matsumoto K, Hirose S, et al. Possible Involvement of Soluble B7-H4 in T Cell-Mediated Inflammatory Immune Responses. Biochem Biophys Res Commun (2009) 389(2):349-53. doi: 10.1016/j.bbrc.2009.08.144

22. Thompson RH, Zang X, Lohse CM, Leibovich BC, Slovin SF, Reuter VE, et al. Serum-Soluble B7x is Elevated in Renal Cell Carcinoma Patients and is Associated With Advanced Stage. Cancer Res (2008) 68(15):6054-8. doi: 10.1158/0008-5472.CAN-08-0869

23. MacGregor HL, Garcia-Batres C, Sayad A, Elia A, Berman HK, Toker A, et al. Tumor Cell Expression of B7-H4 Correlates With Higher Frequencies of Tumor-Infiltrating APCs and Higher CXCL17 Expression in Human Epithelial Ovarian Cancer. Oncoimmunology (2019) 8(12):e1665460. doi: 10.1080/2162402X.2019.1665460

24. Saini M, Szczerba BM, Aceto N. Circulating Tumor Cell-Neutrophil Tango Along the Metastatic Process. Cancer Res (2019) 79(24):6067-73. doi: 10.1158/0008-5472.CAN-19-1972
25. Worzfeld T, Pogge von Strandmann E, Huber M, Adhikary T, Wagner U, Reinartz S, et al. The Unique Molecular and Cellular Microenvironment of Ovarian Cancer. Front Oncol (2017) 7:24. doi: 10.3389/fonc.2017.00024

26. Mego M, Gao H, Cohen EN, Anfossi S, Giordano A, Sanda T, et al. Circulating Tumor Cells (CTC) Are Associated With Defects in Adaptive Immunity in Patients With Inflammatory Breast Cancer. J Cancer (2016) 7(9):1095-104. doi: $10.7150 /$ jca. 13098

27. Hamanishi J, Mandai M, Ikeda T, Minami M, Kawaguchi A, Murayama T, et al. Safety and Antitumor Activity of Anti-PD-1 Antibody, Nivolumab, in Patients With Platinum-Resistant Ovarian Cancer. J Clin Oncol Off J Am Soc Clin Oncol (2015) 33(34):4015-22. doi: 10.1200/JCO.2015.62.3397

28. Brahmer JR, Tykodi SS, Chow LQ, Hwu WJ, Topalian SL, Hwu P, et al. Safety and Activity of Anti-PD-L1 Antibody in Patients With Advanced Cancer. N Engl J Med (2012) 366(26):2455-65. doi: 10.1056/NEJMoa1200694

29. Nicolazzo C, Raimondi C, Mancini M, Caponnetto S, Gradilone A, Gandini O, et al. Monitoring PD-L1 Positive Circulating Tumor Cells in non-Small Cell Lung Cancer Patients Treated With the PD-1 Inhibitor Nivolumab. Sci Rep (2016) 6:31726. doi: 10.1038/srep31726

30. Kulasinghe A, Perry C, Kenny L, Warkiani ME, Nelson C, Punyadeera C. PDL1 Expressing Circulating Tumour Cells in Head and Neck Cancers. BMC Cancer (2017) 17(1):333. doi: 10.1186/s12885-017-3316-3

31. Raimondi L, Raimondi FM, Di Benedetto L, Cimino G, Spinelli GP. PD-L1 Expression on Circulating Tumour Cells May Be Predictive of Response to Regorafenib in Patients Diagnosed With Chemorefractory Metastatic Colorectal Cancer. Int J Mol Sci (2020) 21(18):6907. doi: 10.3390/ ijms 21186907

32. Wang Y, Kim TH, Fouladdel S, Zhang Z, Soni P, Qin A, et al. PD-L1 Expression in Circulating Tumor Cells Increases During Radio(chemo) therapy and Indicates Poor Prognosis in Non-Small Cell Lung Cancer. Sci Rep (2019) 9(1):566. doi: 10.1038/s41598-018-36096-7

33. Papadaki MA, Koutsopoulos AV, Tsoulfas PG, Lagoudaki E, Aggouraki D, Monastirioti A, et al. Clinical Relevance of Immune Checkpoints on Circulating Tumor Cells in Breast Cancer. Cancers (Basel) (2020) 12(2):376. doi: $10.3390 /$ cancers 12020376

34. Iizuka A, Nonomura C, Ashizawa T, Kondou R, Ohshima K, Sugino T, et al. A T-Cell-Engaging B7-H4/CD3-Bispecific Fab-scFv Antibody Targets Human Breast Cancer. Clin Cancer Res an Off J Am Assoc Cancer Res (2019) 25 (9):2925-34. doi: 10.1158/1078-0432.CCR-17-3123

35. Azuma T, Sato Y, Ohno T, Azuma M, Kume H. Serum Soluble B7-H4 is a Prognostic Marker for Patients With non-Metastatic Clear Cell Renal Cell Carcinoma. PloS One (2018) 13(7):e0199719. doi: 10.1371/journal.pone. 0199719

36. Ohki S, Shibata M, Gonda K, Machida T, Shimura T, Nakamura I, et al. Circulating Myeloid-Derived Suppressor Cells are Increased and Correlate to Immune Suppression, Inflammation and Hypoproteinemia in Patients With Cancer. Oncol Rep (2012) 28(2):453-8. doi: 10.3892/or.2012.1812

\section{Conflict of Interest: SK-B is a consultant for Qiagen.}

The remaining authors declare that the research was conducted in the absence of any commercial or financial relationships that could be construed as a potential conflict of interest.

Publisher's Note: All claims expressed in this article are solely those of the authors and do not necessarily represent those of their affiliated organizations, or those of the publisher, the editors and the reviewers. Any product that may be evaluated in this article, or claim that may be made by its manufacturer, is not guaranteed or endorsed by the publisher.

Copyright $\odot 2021$ Mach, Kimmig, Kasimir-Bauer and Buderath. This is an openaccess article distributed under the terms of the Creative Commons Attribution License (CC BY). The use, distribution or reproduction in other forums is permitted, provided the original author(s) and the copyright owner(s) are credited and that the original publication in this journal is cited, in accordance with accepted academic practice. No use, distribution or reproduction is permitted which does not comply with these terms. 